\title{
Application and analysis of the multilevel operating platforms in construction
}

\author{
Qiao Wang ${ }^{1, a^{*}}$, Yanhua $\mathrm{Xu}^{1, \mathrm{~b}}$ and Xiaochen $\mathrm{Li}^{1, \mathrm{c}}$ \\ ${ }^{1}$ Shandong Provincial Academy of Building Research, Jinan, 250031, China \\ awangqiao796@126.com, buyanhua@126.com, ㄷdjjjc@163.com \\ *wangqiao796@126.com
}

Keywords: mast climbing work platform, multilevel operating platforms, external-wall climbing working style

Abstract. This paper analyzes the different demands for construction operating platforms in main structure construction and external decoration. A novel multilevel climbing operating platform applied in main structure construction and external decoration is presented. In addition, an external-wall climbing working style for the multilevel operating platforms is discussed.

\section{Introduction}

Architecture construction is consist of main structure construction and inner \& external decoration. The console scaffold is usually used for low-rise buildings construction and the suspension style scaffold has already found a wide spread application in the higher building construction [1]. After the completion of the main structure construction, the scaffolds mentioned need to be dismantled to start external decoration by means of the suspended access equipment.

The common mast climbing work platforms (MCWP) is shown in Fig.1, which can lift person, materials, equipments to high working place and make several persons work on it [2]. The load capacity of this MCWP is limited, and the free standing height of the mast sections over the last attaching position is less than three meters.

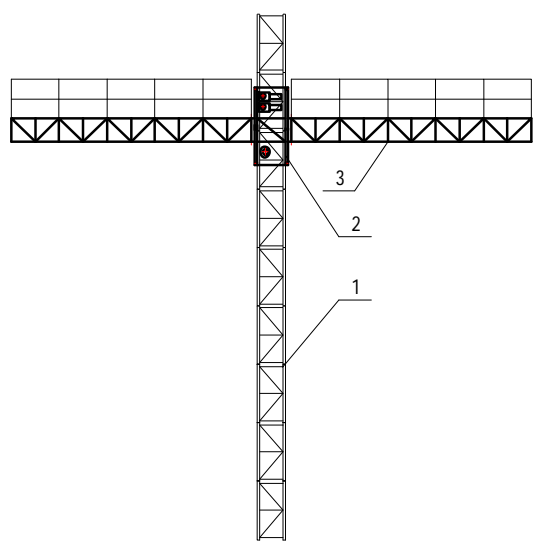

Fig 1. Mast-climbing work platforms

1-Mast

2-Climb frame and working organization

3-Operating platforms

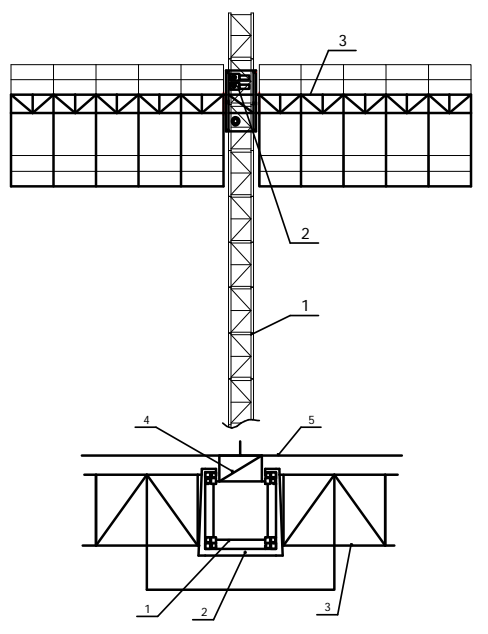

Fig 2. Multilevel operating platforms for the main structure construction and exterior decoration 1 -stand pole guide frame, 2-climb frame and work organization, 3-operating platforms, 4- attaching wall frame, 5 - building

In the phase of main structure construction, especially to the normal concrete main structure configuration, it needs certain time to conserve in order to reach the design intension. If the free 
standing height of the working platforms is less than the height of three layers of the building, it can not fix the attaching wall frame on the main structure with the design intension.

In addition, in the main structure construction phase, the operating platform bears not only the uniform load, but also the centralized load of operators, equipment and tools. Then the common mast-climbing platforms can not satisfy the requirement of the main structure construction. On the other hand, the cost of the mast-climbing platforms is higher than the temporarily installed suspended access equipment. The mast-climbing platforms is rarely used in external wall decoration.

\section{Multilevel operating platforms used to both main structure construction and external decoration}

In order to meet the requirements of the main structure construction and external decoration, a multilevel operating platform is designed, shown in Fig 2. Compared with other work platform, the design has following advantages.

First, climbing frame is closed to help improve its structural rigidity, and there are 16 rollers on the top and bottom of the climbing frame. The rollers spread the load of platform and drive system uniformly to four rails of the mast, which is helpful to reduce the contact strength between rollers and rails. At the same time, the free standing height of the mast sections over the last tie is increased to be suitable for the main structure construction.

Secondly, considering the requirements of the main structure construction[3], the load carrying capacity of the work platform is increased. Single mast platform can bear $600 \mathrm{~kg}$ uniform load. The concentrated load $650 \mathrm{~kg}$ can be placed on the position end of the platform.

Third, the spacing between the levels of multilevel platform can be adjusted according to the construction floor space, which is suitable for the construction personnel to perform their work at the same time in different floors of the building.

Fourth, the length of the platform facade extension is increased according to the changes in the horizontal direction of the building facades.

In the main structure construction, the multilevel operating platforms elevates with the increasing of construction operation layer. It provides operators with the operation platforms and safety protection. After the completion of main structure, it decreases with the building outer surface to finish the external decoration.

\section{A working style of the operating platforms to climb on the external wall of building}

In the assembly of the operating platforms, the masts of the operating platforms are assembled on the top of the base unit, and they are fixed to the building at every intervals by attaching wall frame. When the building is higher, it needs more mast sections and attaching wall frames. To save mast sections and attaching wall frames, a kind of working style is developed for operating platforms to climb on the building' s external wall, shown in Fig 3.

The driving mechanism is fixed on the climb frame, which lifts the operating platforms by the rack and pinion elevation system. In order to realize the function of outer climb, an attaching wall frame is designed, which fixes the climb frame on the building to lift the musts and the operating platforms. In this process, the driving mechanism fixed on the climb frame provides power to lift the musts and the operating platforms. After the climbing completion, the attaching wall frame is dismantled from the climb frame.

To realize the outer climbing function, it is necessary to design a mobile attaching wall device, which can climb on the mast. The configuration and driving mechanism of the mobile attaching device is similar with the climb frame of the operating platforms. In the working condition of the operating platforms, the mobile attaching device ties the mast to the building to make it stable. In the condition the masts and platforms climbing on the external wall, the mobile attaching device is used as a guide 
mechanism of the masts and the operating platforms. When the mobile attaching wall is to climb on the mast, it must be dismantled from the attaching position on the building.

In addition, in the working state of the operating platforms, it needs to design a bottom attaching wall device of the mast, by means of which the first mast is fixed with the building. The bottom

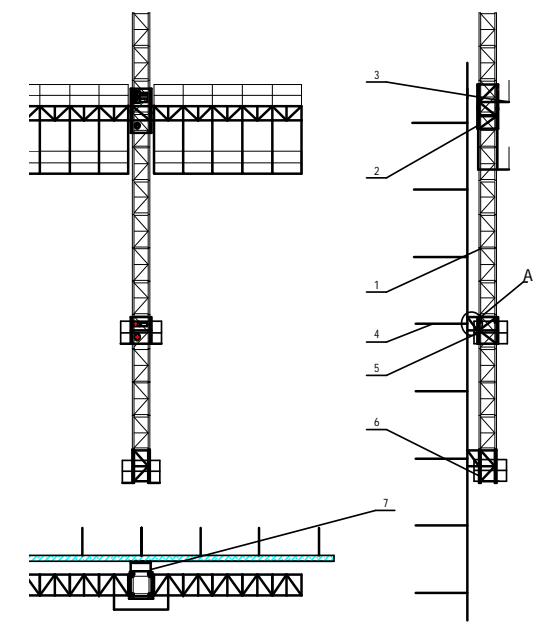

Fig 3. Working style configuration drawing for building operating platforms to construction outer-wall dimming

1-stand pole, 2-climb frame, 3-operating platforms, 4-building

5-moving attaching wall device, 6-bottom attaching wall device

7-attaching wall frame

attaching wall device bears the load of all the masts and working platforms. In the condition the masts and platforms climbing on the external wall, the bottom attaching wall device is dismantled from the building to be lift with the masts. It has a small platform for operators to fix or unfix the attaching wall frame.

Detail implements for operating platforms to climb on the external wall are as follows : 


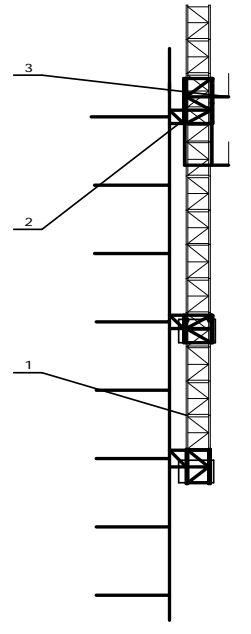

Fig.4-1

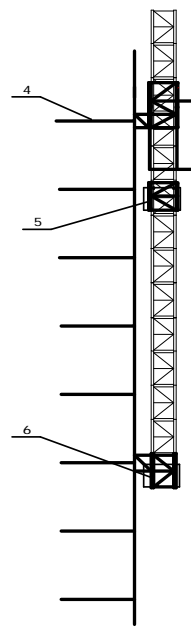

Fig.4-2

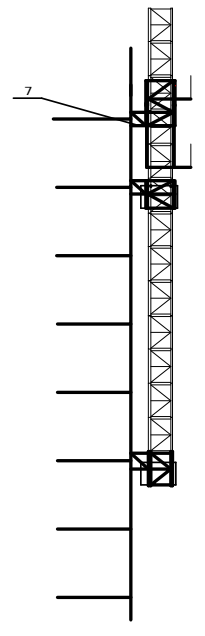

Fig.4-3

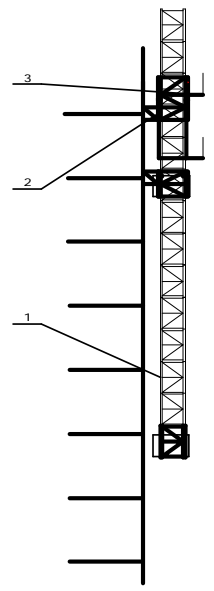

Fig.4-4

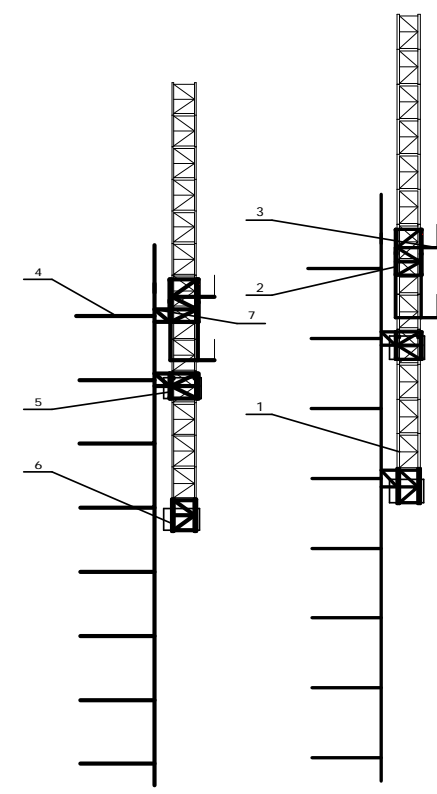

Fig.4-5

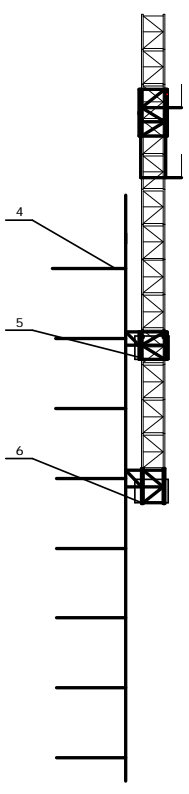

Fig.4-7

The first, in the working condition of the operating platforms, the climb frame and operating platforms climb to the top of the mast. The attaching wall frame is applied to fix the climb frame with the building, shown in Fig 4-1.

The second, the mobile attaching wall device is dismantled from the attaching position on the building to climb two levels to the next attaching wall position, shown in Fig 4-2.

The third, the mobile attaching device is fixed by means of the attaching wall frame on the new attaching wall position, shown in Fig 4-3.

The fourth, the bottom attaching wall device is dismantled from the building to be lift with the masts. So the gravity load of the masts and the platforms is applied on the climb frame by means of the driving mechanism, shown in Fig 4-4.

The fifth, with the aid of the guide device of the climb frame and mobile attaching device, the masts and operating platforms climb on the external wall driven by the rack and pinion elevation system, shown in Fig 4-5.

The sixth, the bottom attaching wall device is fixed on the building by the attaching wall frame, and the climb frame is unfixed from the attaching wall frame, shown in Fig 4-6.

The seventh, the masts and platforms climbing on the external wall is finished. Then the operating platforms can lift person, materials, equipments to high working place, shown in Fig 4-7.

\section{Conclusion}

In this paper a multilevel operating platform is discussed to meet the requirements of the main structure construction and external decoration.

By means of increasing the free standing height above the last attaching position and the bearing load capacity of the operating platforms, the multilevel operating platforms can satisfy the requirement of the main structure and the external decoration. In extra higher building construction, the application of external-wall climbing working style can save a lot of mast sections and reduce the construction cost.

\section{Acknowledgements}

This article supported by the science and technology program of Ministry of Housing and Urban-Rural development (2012-K3-38), research of double level construction operating platforms. 


\section{References}

[1] Du Rong Jun, Practical handbook for building construction scaffold (including vertical convey equipment), China Architecture \& Building Press, Beijing, 1994.05.

[2] GB/T 27547-2011, Elevating work platforms-Mast climbing work platforms, Standard press of China, Beijing, 2012.03

[3] Qin Chun Fang, Wei Zhong Ze, Safety technology handbook for building construction, China Architecture \& Building Press, Beijing, 1991. 